\title{
PET evaluation of light-induced modulation of microglial activation and GLP-1R expression in depressive rats
}

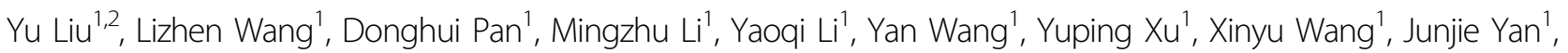 \\ Qiong $\mathrm{Wu}^{1}$, Lin $\mathrm{Lu}^{2,3}$, Kai Yuan ${ }^{3}$ and Min Yang (1) ${ }^{1}$
}

\begin{abstract}
Light therapy has been accepted as a promising therapeutic choice for depression. Positron emission tomography (PET) combined with specific radiotracers has great benefits for revealing pathogenesis and developing therapeutics. This study aimed to investigate the influences of light therapy on microglial activation and glucagon-like peptide-1 receptor (GLP-1R) expression in the brain of depressive rats using $\left[{ }^{18} \mathrm{~F}\right] \mathrm{DPA}-714$ and $\left[{ }^{18} \mathrm{~F}\right]$ exendin-4 PET. The results showed that chronic unpredictable mild stress (CUMS)-induced depressive rats had poorer performance in behavioral tests compared to normal rats $(p<0.05)$ and the depressive-like behavior could be ameliorated by light therapy. Besides, depressive rats had significantly higher $\left[{ }^{18} \mathrm{~F}\right] \mathrm{DPA}-714$ uptake and lower $\left[{ }^{18} \mathrm{~F}\right]$ FDG uptake compare to normal rats in 11 and 9 regions of interest (ROIs) of the brain, respectively $(p<0.05)$. After 5 weeks of light therapy, higher $\left[{ }^{18} \mathrm{~F}\right]$ FDG and $\left[{ }^{18} \mathrm{~F}\right]$ exendin-4 uptake was observed in most ROls of light therapy-treated depressive rats compared to untreated depressive rats $(p<0.05)$ and no significant differences existed in $\left[{ }^{18} \mathrm{~F}\right] \mathrm{DPA}-714$ uptake between the two groups. This study demonstrated that light therapy can ameliorate depressive-like behavior, improve glucose metabolism, and halt the decline of brain GLP-1R expression of depressive rats, but have no effects on microglial activation caused by CUMS. Besides, this study validated that $\left[{ }^{18} \mathrm{~F}\right] \mathrm{DPA}-714$ and $\left[{ }^{18} \mathrm{~F}\right]$ exendin-4 PET have the potential for noninvasive evaluation of microglial activation and GLP-1R expression in the brain of depression.
\end{abstract}

\section{Introduction}

In the past few years, light therapy has been accepted as an optional therapeutic choice for some neuropsychiatric disorders such as major depressive disorder, circadian phase sleep disorder, Parkinson's disease, and Alzheimer's disease $^{1-5}$. However, the mechanisms of light therapy for depression remain unclear and there is a lack of efficacy evaluation methods for this treatment. Positron emission

\footnotetext{
Correspondence: Kai Yuan (yuankai@pku.edu.cn) or

Min Yang (yangmin@jsinm.org)

${ }^{1} \mathrm{NHC}$ Key Laboratory of Nuclear Medicine, Jiangsu Key Laboratory of Molecular Nuclear Medicine, Jiangsu Institute of Nuclear Medicine, Wuxi 214063 Jiangsu, China

${ }^{2}$ Peking-Tsinghua Center for Life Sciences, Peking University, 100871 Beijing, China

Full list of author information is available at the end of the article

These authors contributed equally: Yu Liu, Lizhen Wang
}

tomography (PET) is a well-recognized imaging method for noninvasive diagnosis and efficacy evaluation of neuropsychiatric disorders, besides, PET combined with specific radiotracers has great benefits for revealing pathogenesis and developing therapeutics. For example, recent studies demonstrated the reduced cerebral MAOA levels and serotonin transporter binding potential in patients with depression after a few weeks of bright light therapy using $\left[{ }^{11} \mathrm{C}\right]$ harmine and $\left[{ }^{11} \mathrm{C}\right] \mathrm{DASB}$ PET imaging $^{6,7}$. However, the complex pathology of depression makes it still possesses many other important targets worth exploring.

The inflammatory hypothesis in depression has received lots of attention in recent years. Numerous studies illustrated that depression may be associated with microglial activation and neuroinflammation ${ }^{8,9}$. However, whether

\section{(c) The Author(s) 2021}

(c) (i) Open Access This article is licensed under a Creative Commons Attribution 4.0 International License, which permits use, sharing, adaptation, distribution and reproduction cc) in any medium or format, as long as you give appropriate credit to the original author(s) and the source, provide a link to the Creative Commons license, and indicate if changes were made. The images or other third party material in this article are included in the article's Creative Commons license, unless indicated otherwise in a credit line to the material. If material is not included in the article's Creative Commons license and your intended use is not permitted by statutory regulation or exceeds the permitted use, you will need to obtain permission directly from the copyright holder. To view a copy of this license, visit http://creativecommons.org/licenses/by/4.0/. 
light therapy has effects on neuroinflammation in depression remains controversial ${ }^{10,11}$. The $18 \mathrm{kDa}$ translocator protein (TSPO) found on the outer mitochondrial membrane has been considered to be a reliable biomarker of neuroinflammation because its expression is low in healthy brain under physiological conditions, but significantly elevated in activated microglia under inflammatory $^{12}$. Over the past three decades, dozens of radioligands specifically target TSPO have been synthesized and some of them were tested in preclinical and clinical studies of depression ${ }^{13-17} \cdot\left[{ }^{18} \mathrm{~F}\right] \mathrm{DPA}-714$ is a second-generation TSPO radiotracer that has higher signal-to-noise ratio and lower nonspecific uptake in the brain compared to the currently widely used $\left[{ }^{11} \mathrm{C}\right]$ PK11195 ${ }^{18}$. Besides, ${ }^{18} \mathrm{~F}$ has a longer half-life $(109.8 \mathrm{~min})$ compared to ${ }^{11} \mathrm{C}$ (20.4 min) which is more suitable for clinical application. Due to its promising properties, $\left[{ }^{18} \mathrm{~F}\right]$ DPA-714 has been investigated in many pathological conditions including stroke ${ }^{19}$, epilepsy ${ }^{20}$, and Alzheimer's disease $^{21}$. However, few studies have explored its potential in depression. Our previous study suggested that rats with depression might have elevated $\left[{ }^{18} \mathrm{~F}\right] \mathrm{DPA}-714$ uptake in the hippocampus, but the more comprehensive uptake profile and imaging performance of $\left[{ }^{18} \mathrm{~F}\right] \mathrm{DPA}-714 \mathrm{PET}$ in the whole brain of depression need to be further investigated $^{22}$.

Accumulating data suggested that altered gut microbiota could have influences in psychiatric disorders ${ }^{23}$. The glucagon-like peptide-1 (GLP-1), a 30-amino acid peptide hormone which is mainly produced by intestine L cells, has been considered to be a mediator in the bidirectional microbiota-gut-brain axis ${ }^{24}$. Several studies have demonstrated that GLP-1 receptor (GLP-1R) agonists share some key enzymes and signaling pathways with antipsychotic drugs ${ }^{25-27}$ and a recent meta-analysis described the antidepressant effects of GLP-1R agonists $^{28}$. Besides, studies also showed that GLP-1R agonists as well as some probiotics could improve depressive behaviors by upregulating GLP-1R expression in the brain $^{29,30}$. However, no studies have explored whether light therapy can mediate cerebral GLP-1R expression. Besides, a handful of studies have evaluated the distribution and expression of GLP-1R in the central nervous system using immunohistochemistry ${ }^{31}$. However, no reliable imaging methods have been developed for the noninvasive visualization and quantification of brain GLP$1 \mathrm{R}$ expression. In our previous study, we firstly evaluated the feasibility of GLP-1R PET in the brain using $\left[{ }^{18} \mathrm{~F}\right] \mathrm{AlF}$ NOTA-MAL-Cys ${ }^{39}$-exendin-4 $\left(\left[{ }^{18}\right.\right.$ F $]$ exendin-4) and demonstrated the influences of age on GLP-1R expres$\operatorname{sion}^{32}$. However, a comprehensive evaluation in the performance of GLP-1R PET should be conducted and more adequate imaging parameters should be provided to prove that GLP-1R PET can be used for brain imaging in specific pathology and provide a reference for further improvement of this imaging method.

In summary, this study aimed to investigate whether chronic unpredictable mild stress (CUMS)-induced depression rat model has altered microglial activation and brain GLP-1R expression using PET imaging and describe the imaging patterns of specific radiotracers $\left[{ }^{18} \mathrm{~F}\right] \mathrm{DPA}$ 714 and $\left[{ }^{18} \mathrm{~F}\right]$ exendin- 4 in depressive rats. Furthermore, we would like to explore whether microglial activation and brain GLP-1R expression could be mediated by light therapy in depressive rats using these PET imaging methods. Here, we would additionally use $\left[{ }^{18}\right.$ F]FDG PET as a reference, which is used for the evaluation of glucose metabolism and is the most widely accepted and used PET tracer in the clinic ${ }^{33}$.

\section{Materials and methods \\ Animals}

Sprague-Dawley rats (male, 5-6 weeks, 250-300 g) used in this study were purchased from Changzhou Cavens Laboratory Animal Co., Ltd. (Changzhou, China) and housed in the Laboratory Animal Center of Jiangsu Institute of Nuclear Medicine under a temperaturecontrolled $\left(23 \pm 2{ }^{\circ} \mathrm{C}\right)$ and humidity-controlled $(\sim 50 \%)$ condition. Rats were free to access food and water and the room was in a $12 \mathrm{~h}$ light/dark cycle $(7: 00$ a.m. to 7:00 p.m.). Sample size was determined based on previous published data as sufficient to obtain statistical significance. All animal experiments involved in this study were approved by the Laboratory Animal Management and Ethics Committee of Jiangsu Institute of Nuclear Medicine.

\section{CUMS protocol}

Rats were randomly selected for modeling. The CUMS protocol was performed to induce a rodent model of depression $^{34}$. Briefly, rats were housed individually and exposed to one of the following stressors: food deprivation for $24 \mathrm{~h}$; water deprivation for $24 \mathrm{~h}$; clamp the tail for $5 \mathrm{~min}$; overnight illumination; swimming in cold water $\left(4^{\circ} \mathrm{C}\right)$ for $5 \mathrm{~min}$; ultrasound stimulation $(110 \mathrm{~dB})$ for $1 \mathrm{~h}$; tilt the cage at $45^{\circ}$ for $12 \mathrm{~h}$; hot temperature $\left(45^{\circ} \mathrm{C}\right)$ for $5 \mathrm{~min}$; cage shaking ( $1 \mathrm{time} / \mathrm{s})$ for $15 \mathrm{~min}$; electrical stimulation $(1 \mathrm{~mA}, 30 \mathrm{~V}, 30 \mathrm{~s}$ stimulation with $1 \mathrm{~min}$ interval) on the sole for $5 \mathrm{~min}$; wet environment for $12 \mathrm{~h}$. The stressor given each day was random and the whole protocol lasted for 4 weeks.

\section{Behavioral tests}

The behavioral tests were performed according to previous studies ${ }^{35}$. For sucrose preference test (SPT), rats were trained to adapt and learn to drink $1 \%$ sucrose solution. Then, all rats were given two bottles at the same time: one bottle of $1 \%$ sucrose water and one bottle of tap 
water. The positions of the two bottles were changed every $12 \mathrm{~h}$. The sucrose water and tap water consumed every $24 \mathrm{~h}$ were recorded and sucrose preference was calculated as sucrose preference $(\%)=$ sucrose water consumption/(sucrose water + tap water consumption). For forced swimming test (FST), rats were placed in a transparent cylindrical bucket of water (depth of $35-40 \mathrm{~cm}$, diameter of $30 \mathrm{~cm}$, and temperature of $\left.22-24^{\circ} \mathrm{C}\right)$. The immobile time of each rat in the water was blindly recorded by two independent observers. The total duration is $6 \mathrm{~min}$ and the total immobility was calculated using the data of the last $5 \mathrm{~min}$. For open field test (OFT), rats were placed in a customized box $(50 \mathrm{~cm} \times 50 \mathrm{~cm} \times$ $45 \mathrm{~cm}$ ) in a room with dim light. The movement of rats within 5 min was recorded with an infrared camera and the total movement distance was measured. Rats induced by CUMS and verified by behavioral tests to have depressive-like behaviors were included as depression rats (CUMS group), otherwise they would be excluded.

\section{Radiochemistry}

The radiolabeling precursor DPA-714 was generously donated by Professor Mengchao Cui of Beijing Normal University. Cys ${ }^{39}$-exendin- 4 was purchased from Apeptide Co., Ltd. (Shanghai, China). NOTA-MAL was purchased from CheMatech (Dijon, France). The $\left[{ }^{18} \mathrm{~F}\right] \mathrm{FDG}$ was provided by Wuxi Fourth People's Hospital and had satisfied the clinical standard. The ${ }^{18} \mathrm{~F}^{-}$was produced on the cyclotron (Sumitomo Heavy Industries, Japan) of Jiangsu Institute of Nuclear Medicine.

The radiosynthesis of $\left[{ }^{18} \mathrm{~F}\right] \mathrm{DPA}-714$ and $\left[{ }^{18} \mathrm{~F}\right]$ exendin- 4 was conducted as described previously with some modifications ${ }^{36,37}$. Briefly, for $\left[{ }^{18} \mathrm{~F}\right] \mathrm{DPA}-714$, the ${ }^{18} \mathrm{~F}^{-}$ produced by the cyclotron was captured on a QMA cartridge (Waters, USA) and eluted into a $5 \mathrm{~mL}$ reaction vessel by $1.5 \mathrm{~mL} \mathrm{~K}_{2.2 .2} / \mathrm{K}_{2} \mathrm{CO}_{3}$. The mixed solution was dried three times under nitrogen flow at $100^{\circ} \mathrm{C}$ wherein $1.5 \mathrm{ml}$ of extra-dry acetonitrile (Acros Organics, Belgium) was added each time. Then, $3 \mathrm{mg}$ of DPA-714 stored under vacuum was dissolved in $0.6 \mathrm{ml}$ of extra-dry acetonitrile and added to the completely dried ${ }^{18} \mathrm{~F}^{-}$and reacted at $97^{\circ} \mathrm{C}$ for $10 \mathrm{~min}$. The reacted solution was cooled to room temperature and injected into a semipreparative HPLC (Waters, USA). The mobile phase was deionized water with $0.1 \%$ triethylamine (SCR, China) and acetonitrile (SCR, China) with $0.1 \%$ triethylamine (50/50, $\mathrm{v} / \mathrm{v})$ and the flow rate was $5 \mathrm{~mL} / \mathrm{min}$. The collected $\left[{ }^{18} \mathrm{~F}\right]$ DPA-714 fraction was injected into a C18 cartridge (Waters, USA) and then eluted using $0.6 \mathrm{ml}$ of ethanol (SCR, China) to get the final product. The entire synthesis process could be completed within $1 \mathrm{~h}$ while the radiochemical purity is $\geq 99 \%$ and the yield is $\sim 30 \%$. For $\left[{ }^{18} \mathrm{~F}\right]$ exendin-4, the NOTA-MAL-Cys ${ }^{39}$-exendin-4 synthesized according to the previous study ${ }^{37}$ was dissolved in deionized water, and then the $\mathrm{AlCl}_{3}$ solution, acetonitrile, and glacial acetic acid (Sigma-Aldrich, USA) was added in order, finally the ${ }^{18} \mathrm{~F}^{-}$solution was added, then the reaction was conducted at $100{ }^{\circ} \mathrm{C}$ for $10 \mathrm{~min}$. The product was captured onto a $\mathrm{C} 18$ cartridge using sterile water and finally rinsed off with $\mathrm{HCl}$-containing ethanol. The entire process could be finished within $30 \mathrm{~min}$ with a radiochemical purity $>95 \%$ and yield around $25 \%$.

\section{PET scans}

PET scans were conducted on an Inveon microPET scanner (Siemens Medical Solutions, Germany) and the protocol was based on previous studies ${ }^{19,20}$. Rats were anesthetized with the isoflurane $/ \mathrm{O}_{2}$ mixture (induction, $4 \%$; maintenance, $2-2.5 \%)$. For $\left[{ }^{18} \mathrm{~F}\right] \mathrm{FDG}$, rats were fasted one night before PET scan. The 10 min static images were acquired at $60 \mathrm{~min}$ after intravenous injection of $\left[{ }^{18} \mathrm{~F}\right]$ DPA-714 or $\left[{ }^{18} \mathrm{~F}\right] \mathrm{FDG}$, and $30 \mathrm{~min}$ after injection of $\left[{ }^{18} \mathrm{~F}\right]$ exendin-4, respectively. To obtain dynamic PET images of $\left[{ }^{18} \mathrm{~F}\right] \mathrm{DPA}-714$ and $\left[{ }^{18} \mathrm{~F}\right]$ exendin- 4 , four depressive rats were randomly selected and the image acquisitions were continued from the start of the injection to $60 \mathrm{~min}$ after injection. For the blocking experiments, blocking dose $\left(5 \mathrm{mg} / \mathrm{kg}\right.$ body weight) of unlabeled DPA-714 and Cys ${ }^{39}$. exendin-4 were intravenously injected $10 \mathrm{~min}$ before the dynamic acquisitions. The dynamic data were sorted into 31 frames $(30 s \times 10,60 s \times 5,120 s \times 10,300 s \times 6)$. Images were normalized, corrected, and reconstructed using Fourier rebinning (FORE) and 3-dimensional ordered subsets expectation maximum (OSEM 3D) algorithm $(128 \times 128$ matrix size, 2 OSEM iterations, and no scatter correction).

\section{Imaging data analysis}

PMOD software was used for all image processing. PET images were co-registered to the anatomical data of the MRI rat brain template provided by PMOD. Thirty-three regions of interest (ROIs) in the brain were drawn automatically. The standard uptake value (SUV) was calculated for static images. For dynamic images, SUV was calculated for each ROI of all frames, and the nondisplaceable binding potential $\left(\mathrm{BP}_{\mathrm{ND}}\right)$ of each $\mathrm{ROI}$ was generated with SRTM2 using the normalized data from blocking group $\left(\mathrm{BP}_{\mathrm{ND}}-\mathrm{BLO}\right)$ and cerebellum gray matter $\left(\mathrm{BP}_{\mathrm{ND}}-\mathrm{CBL}\right)$ as the pseudo-reference region, respectively. Parametric images were also derived using $\mathrm{BP}_{\mathrm{ND}}-\mathrm{BLO}$ and $\mathrm{BP}_{\mathrm{ND}}-\mathrm{CBL}$.

\section{Western blot}

The rats' hippocampi tissue was homogenized in RIPA lysis buffer (Beyotime, China). Protein samples were run on $12 \%$ Bis-Tris gels (Invitrogen, USA) and transferred to PVDF membranes (Beyotime, China). Primary antibodies against anti-Iba-1 (1:200, Abcam, USA), anti-IL-1 $\beta$ 
(1:1000, ABclonol, China), anti-IL-6 (1:1000, ABclonol, China), anti-TNF- $\alpha$ (1:200, ABclonol, China), anti-GLP1R (1: 1000, GeneTex, USA), and anti- $\beta$-actin (1:1000, Beyotime, China) were incubated overnight at $4{ }^{\circ} \mathrm{C}$. Secondary antibodies (1:1000-1:10,000, Beyotime, China) were incubated for $2 \mathrm{~h}$ at room temperature.

\section{Immunofluorescence}

The brain tissue of rats was taken out after perfusion and made into $12 \mu \mathrm{m}$ frozen sections. Sections were permeabilized and blocked in PBS containing Triton X-100 and serum. Sections then were incubated with primary antibodies overnight at $4{ }^{\circ} \mathrm{C}$. The primary antibodies including anti-Iba-1 (1:100, Abcam, USA), anti-IL$1 \beta$ (1:100, ABclonol, China), anti-IL-6 (1:100, ABclonol, China), and anti-GLP-1R (1:100, GeneTex, USA). Secondary antibodies (1:1000, ThermoFisher, USA) were used for sections for $1 \mathrm{~h}$ in the dark. Sections were finally observed with an Olympus fluorescent inverted microscope.

\section{Light therapy protocol}

Rats were randomly selected for light therapy (LT group). Briefly, rats were placed in transparent cages in customized opaque boxes. Blue light with a dominant wavelength of $460.1 \mathrm{~nm}^{38}$ and illuminance of $3000 \mathrm{lux}^{35}$ was applied. Besides, we installed a frequency regulator to stabilize the light at $40 \mathrm{~Hz}$ and also installed heat sinks on the box. Five weeks of uninterrupted light therapy protocol were performed for $3 \mathrm{~h}$ every day (8:00 a.m.-11:00 a. $\mathrm{m}$.), and the rats could still get food and water freely during treatment.

\section{Statistical analysis}

Data were expressed as mean \pm SD and statistical analysis was accomplished using Stata/SE 12.0. Student $t$ test was used in behavioral tests before light therapy, PET imaging analysis at baseline, and WB results. ANOVA with Bonferroni correction was used in behavioral tests and PET imaging analysis during and after light therapy. Pearson correlation coefficient was used to assess the correlation between $\mathrm{SUV}, \mathrm{BP}_{\mathrm{ND}}-\mathrm{BLO}$, and $\mathrm{BP}_{\mathrm{ND}}-\mathrm{CBL}$. Significance levels were set at $p<0.05$. Data were plotted using Origin 9.0.

\section{Results}

\section{Depressive-like behavior was induced by CUMS and} ameliorated by light therapy

During the modeling of CUMS, the body weight of CUMS rats showed a tendency to decrease. In contrast, the body weight of the rats in control group increased in fluctuation (Fig. 1b). After 4 weeks of modeling, CUMS rats showed a much longer total immobility time in the FST and a much shorter movement distance in the OFT compared to control group (Fig. 1c, d). Besides, the SPT results demonstrated that CUMS would lead to a slight but significant reduction of sucrose preference (Fig. 1e). During the treatment of light therapy, the body weight of the rats in LT group gradually increased and approached the control group, while the body weight of the untreated CUMS group decreased in fluctuation (Fig. 1f). As shown in Fig. $1 \mathrm{~g}-\mathrm{i}$, after 2 weeks of light therapy, the total immobility time of LT group in the FST was almost the same as that of control group while the CUMS group showed no amelioration (Fig. 1g). Although the total movement distance of LT group in the OFT was still significantly different from control group, it has shown an increasing tendency and also a significant difference between LT and CUMS group (Fig. 1h). The results of the SPT showed close sucrose preference between LT and control group, and both of them were significantly higher than that of CUMS group (Fig. 1i). Besides, Fig. 1g-i showed that light therapy for 5 weeks or 2 weeks have similar effects on CUMS-induced depressive behavior.

\section{PET imaging analysis at baseline}

Time-activity curves representing the radioactive uptake of $\left[{ }^{18} \mathrm{~F}\right] \mathrm{DPA}-714$ and $\left[{ }^{18} \mathrm{~F}\right]$ exendin- 4 in the brain were shown in Fig. 2. After bolus injection, both these two tracers reached their highest accumulation rapidly and decreased thereafter. The reduction of $\left[{ }^{18} \mathrm{~F}\right]$ exendin- 4 uptake was faster than that of $\left[{ }^{18} \mathrm{~F}\right] \mathrm{DPA}-714$. After preinjection of non-radiolabeled precursor, the brain uptake of the two radiotracers quickly fell after reaching the peak, and the decrease in the uptake of the two radiotracers in the brain of the unblocked groups was significantly slower than that of blocked groups.

The $\mathrm{BP}_{\mathrm{ND}}-\mathrm{CBL}$ and $\mathrm{BP}_{\mathrm{ND}}-\mathrm{BLO}$ results of $\left[{ }^{18} \mathrm{~F}\right] \mathrm{DPA}-714$ and $\left[{ }^{18} \mathrm{~F}\right]$ exendin- 4 in 33 ROIs of CUMS rats calculated by SRTM2 were shown in Supplementary Table S1. Both $\mathrm{BP}_{\mathrm{ND}}-\mathrm{CBL}$ and $\mathrm{BP}_{\mathrm{ND}}-\mathrm{BLO}$ correlated well with SUV for $\left[{ }^{18} \mathrm{~F}\right] \mathrm{DPA}-714$ and $\left[{ }^{18} \mathrm{~F}\right]$ exendin-4, and good correlations could also be found between $\mathrm{BP}_{\mathrm{ND}}-\mathrm{CBL}$ and $\mathrm{BP}_{\mathrm{ND}}-\mathrm{BLO}$ (Supplementary Table S2). For $\left[{ }^{18} \mathrm{~F}\right] \mathrm{DPA}-714$, images derived from $\mathrm{BP}_{\mathrm{ND}}-\mathrm{BLO}$ showed more detailed radioactive uptake distribution compared to images derived from $\mathrm{BP}_{\mathrm{ND}}-\mathrm{CBL}$, which was consistent with quantified data. For $\left[{ }^{18} \mathrm{~F}\right]$ exendin-4, images derived from $\mathrm{BP}_{\mathrm{ND}}-\mathrm{CBL}$ and $\mathrm{BP}_{\mathrm{ND}}-\mathrm{BLO}$ both showed low uptake profile in the brain, which was also consistent with quantified data, however, the limited visual image quality of $\left[{ }^{18} \mathrm{~F}\right]$ exendin4 indicated that the visualization capability for this tracer needs to be further improved (Supplementary Fig. S1).

\section{Multimodal assessment of CUMS-induced depressive rats ${ }^{18}$ F]DPA-714 PET}

As shown in Fig. 3a, after the modeling period, CUMS rats had significant higher $\left[{ }^{18} \mathrm{~F}\right] \mathrm{DPA}-714$ SUV compare to 


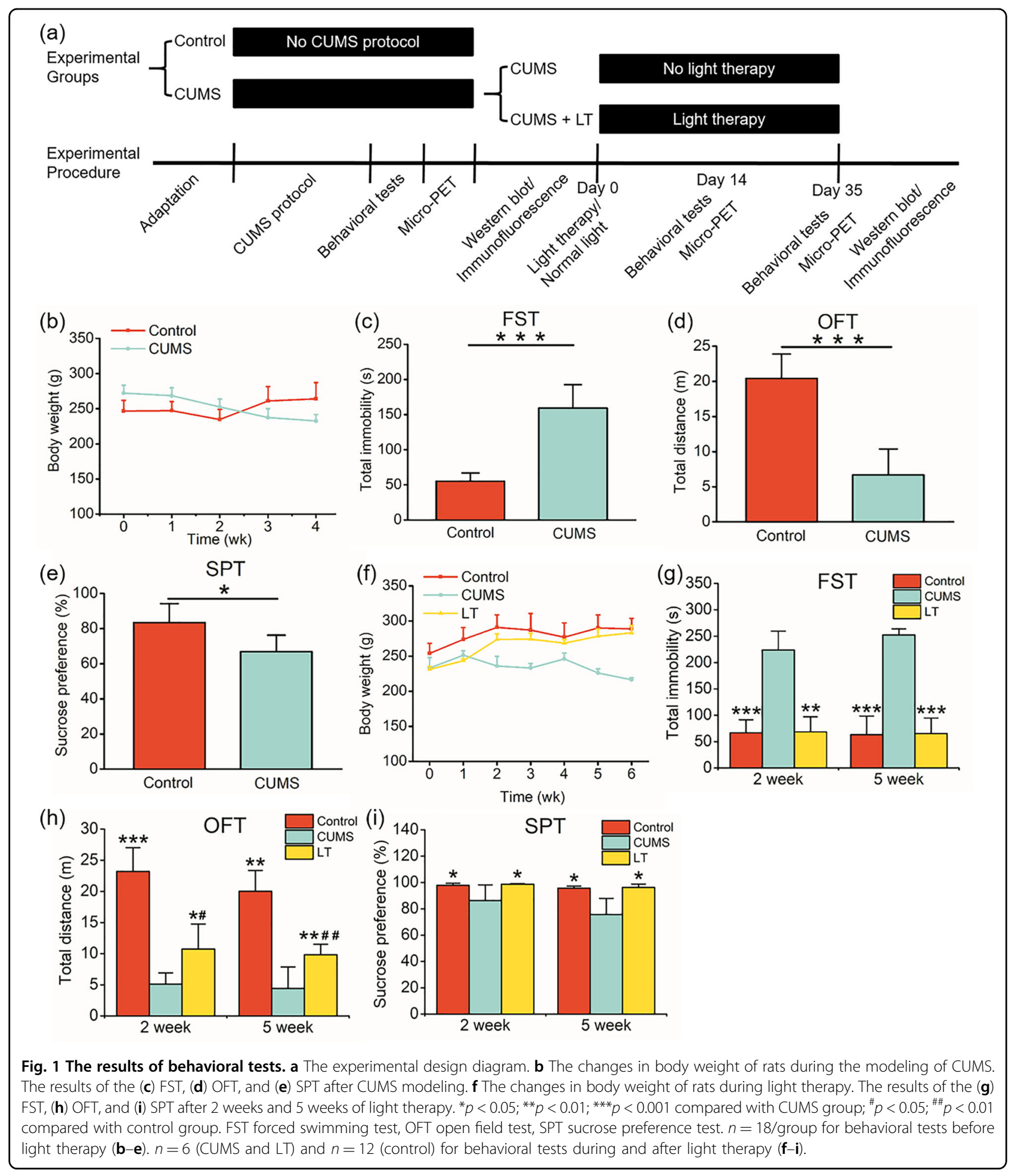

normal rats in 11 ROIs of the brain including amygdala, auditory cortex, cingulate cortex, motor cortex, parietal cortex, visual cortex, posterior hippocampus, hypothalamus, midbrain, ventral tegmental area, and pons $(p<0.05)$. Besides, although there were no significant differences, the $\left[{ }^{18} \mathrm{~F}\right] \mathrm{DPA}-714$ uptake in most other brain regions of depressive rats was higher than that of normal rats, suggesting that depression would induce extensive microglial activation in the brain (Supplementary Table S3). 

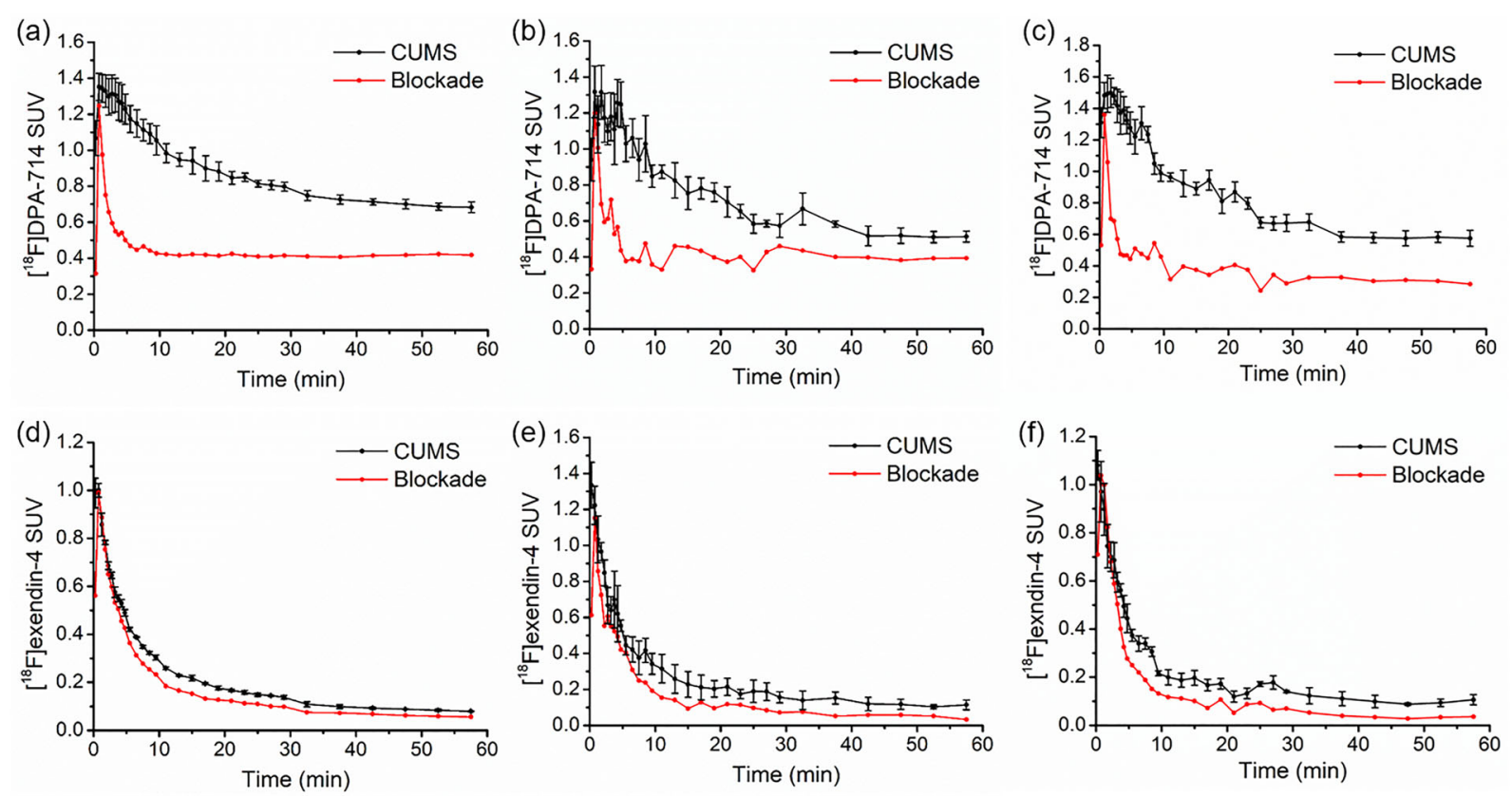

Fig. 2 Dynamic PET analysis and blocking experiments of $\left[{ }^{18} \mathrm{~F}\right] \mathrm{DPA}-714$ and $\left[{ }^{18} \mathrm{~F}\right]$ exendin- 4 in the brain of depressive rats. Time-activity curves of $\left[{ }^{18} \mathrm{~F}\right] \mathrm{DPA}-714$ uptake in whole brain (a), posterior hippocampus (b), and medial prefrontal cortex (c). Time-activity curves of $\left[{ }^{18} \mathrm{~F}\right]$ exendin-4 uptake in whole brain (d), hypothalamus (e), and ventral tegmental area $(\mathbf{f}) . n=4$ for each group of dynamic experiments and $n=1$ for each group of blocking experiments.

\section{$\left[{ }^{18}\right.$ F]exendin-4 PET}

As shown in Supplementary Table S3, there were no significant differences in $\left[{ }^{18} \mathrm{~F}\right]$ exendin- 4 uptake in most brain regions between CUMS and normal rats. However, the interesting higher accumulation of $\left[{ }^{18} \mathrm{~F}\right]$ exendin- 4 in auditory cortex and pituitary of CUMS rats than normal rats were found.

\section{$\left[{ }^{18}\right.$ F]FDG PET}

The widespread lower accumulation of $\left[{ }^{18} \mathrm{~F}\right] \mathrm{FDG}$ was observed in the brain of CUMS rats compared to normal rats (Supplementary Table S3), and significant differences existed in the whole brain, auditory cortex, insular cortex, motor cortex, parietal cortex, retrosplenial cortex, somatosensory cortex, visual cortex, and pons, indicating that CUMS-induced depression would lead to an extensive decline of glucose metabolism in the brain (Fig. 3b).

\section{Western blot and immunofluorescence}

Then, we evaluated the Iba- 1 and inflammatory factors in the hippocampus using WB and IF. The results of WB showed elevated Iba-1, IL-6, IL-1 $\beta$, and TNF- $\alpha$ levels in hippocampus of CUMS rats compared to normal rats (Fig. 3c). We also detected the higher expression of Iba-1, IL-6, and IL- $1 \beta$ in the hippocampus of CUMS rats using IF (Fig. 3d, Supplementary Fig. S2). These results were consistent with the quantification of PET imaging.

\section{Multimodal evaluation of light therapy for CUMS-induced depression $\left[^{18}\right.$ F]DPA-714 PET}

After 2 weeks of light therapy, no significant differences in $\left[{ }^{18} \mathrm{~F}\right] \mathrm{DPA}-714$ uptake existed among LT treated CUMS rats, untreated CUMS rats, and CUMS rats before LT (baseline) in all 33 ROIs (Supplementary Table S4). The same results were also found at 5 weeks after light therapy (Supplementary Table S5).

\section{$\left[{ }^{18}\right.$ F]exendin-4 PET}

As shown in Supplementary Table S4, after 2 weeks of light therapy, there were no significant differences in $\left[{ }^{18} \mathrm{~F}\right]$ exendin-4 uptake in all 33 ROIs of the brain between LT group and untreated CUMS group. Besides, neither of these two groups showed any significant differences compared to baseline. After 5 weeks of light therapy, significant lower uptake of $\left[{ }^{18} \mathrm{~F}\right]$ exendin-4 was observed in untreated CUMS group compared to LT group in ten ROIs $(p<0.05)$. Besides, the $\left[{ }^{18} \mathrm{~F}\right]$ exendin- 4 uptake in five ROIs of untreated CUMS group was significantly lower than that of baseline. However, no significant differences in the $\left[{ }^{18} \mathrm{~F}\right]$ exendin- 4 uptake could be quantified in any ROI between LT group and baseline (Fig. 4a and Supplementary Table S5). These results suggested that light therapy prevented the decrease of GLP-1R expression in the brain of CUMS-induced depressive rats. 


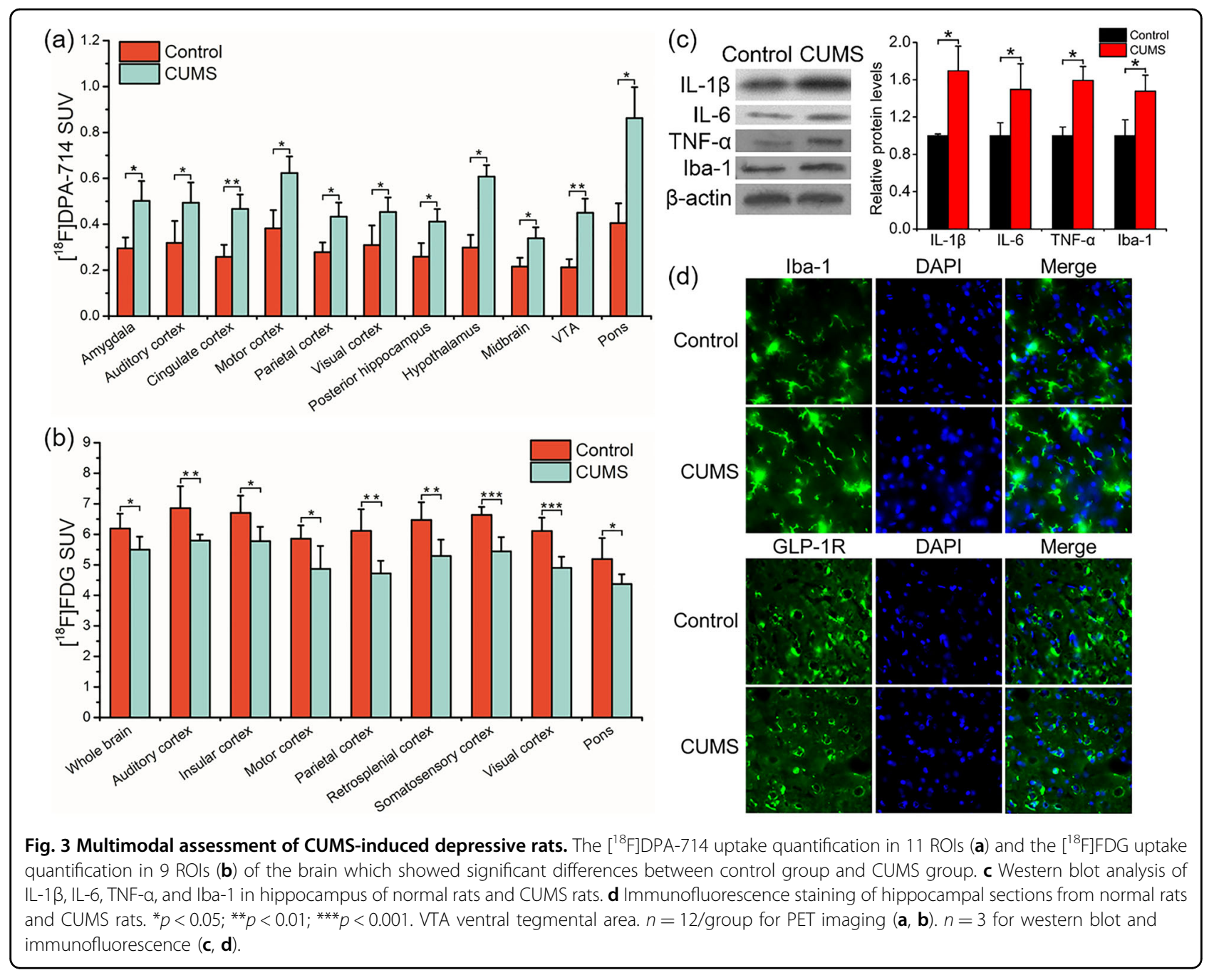

\section{$\left[{ }^{18}\right.$ FJFDG PET}

After 2 weeks of light therapy, although there were no significant differences, the $\left[{ }^{18} \mathrm{~F}\right] \mathrm{FDG}$ uptake in most ROIs of untreated CUMS group was lower than that of LT group. Besides, significantly lower uptake of $\left[{ }^{18} \mathrm{~F}\right] \mathrm{FDG}$ existed in ten ROIs of untreated CUMS group compared to baseline while significant different $\left[{ }^{18} \mathrm{~F}\right] \mathrm{FDG}$ uptake between LT group and baseline could only be observed in auditory cortex (Fig. 4b and Supplementary Table S4). After 5 weeks of light therapy, the $\left[{ }^{18} \mathrm{~F}\right] \mathrm{FDG}$ uptake in 24 ROIs of untreated CUMS group was significantly lower than that of LT group, besides, the $\left[{ }^{18} \mathrm{~F}\right]$ FDG uptake in $20 \mathrm{ROIs}$ of LT group was significantly higher than that of baseline. These results indicated that light therapy could reverse the extensive decreased glucose metabolism in the brain caused by CUMS (Fig. 4c and Supplementary Table S5).

\section{Western blot and immunofluorescence}

Since we observed a decreased $\left[{ }^{18} \mathrm{~F}\right]$ exendin- 4 uptake in the brain of untreated CUMS group compared to LT group, we validated the different expression of GLP-1R in the hippocampus between the two groups using WB and IF (Fig. 4d, e). Besides, IF staining of Iba-1 and inflammatory factors in hippocampal sections showed no obvious differences between CUMS group and LT group (Fig. 4d and Supplementary Fig. S3).

\section{Discussion}

Recent studies confirmed that neuroinflammation plays an important role in the pathological process of depression. The evidence of microglial activation and neuroinflammation found in postmortem further supported this view $^{9,39}$. PET as a functional imaging technique that has been widely used in neuropsychiatric disorders can visualize specific pathological processes noninvasively, dynamically, comprehensively, and has excellent quantitative capability ${ }^{40}$. In recent years, radiotracers targeting TSPO have been used in various neuropsychiatric disorders. Several clinical trials proved that these radiotracers have promising quantification ability for microglial 


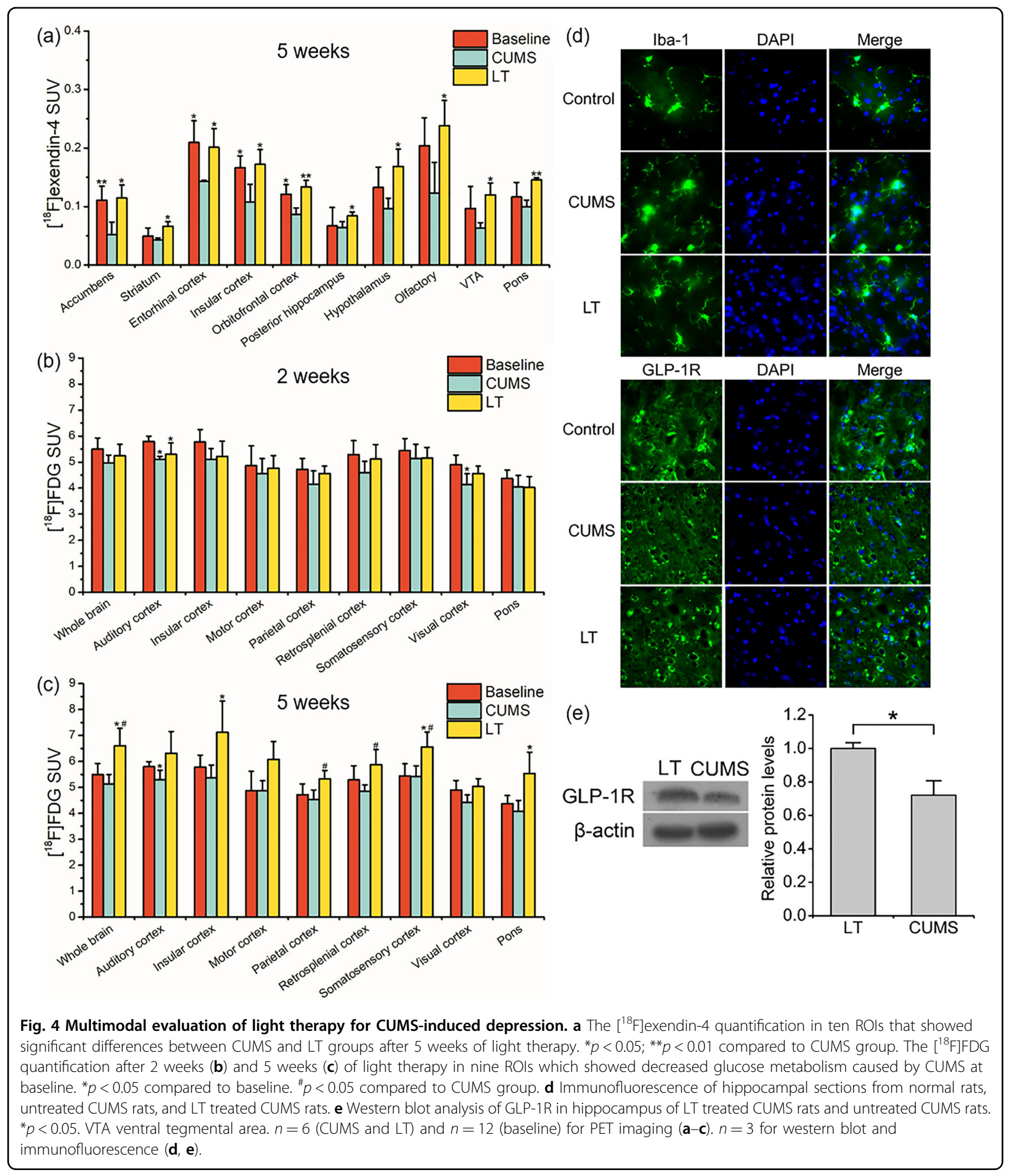

activation in depression ${ }^{13-15,17}$. Our previous study proved for the first time that $\left[{ }^{18} \mathrm{~F}\right] \mathrm{DPA}-714$ detected microglial activation in the hippocampus of depression rats $^{22}$. In this present study, we further confirmed the neuroinflammatory changes induced by CUMS in multiple brain regions using $\left[{ }^{18} \mathrm{~F}\right] \mathrm{DPA}-714$ PET. On the other hand, pathological experiments such as WB and IF are difficult to reflect the neuroinflammation in various regions of the brain at the same time. In this study, we subdivided the PET images of the whole brain into 32 
regions and calculated the SUV for each ROI, thus obtained a relatively comprehensive whole brain inflammation profile.

Another primary strength of PET is its excellent quantification ability ${ }^{41}$. However, due to the small size of rodent brain and the wide expression of TSPO, the quantification of TSPO PET has always been a challenge ${ }^{42}$. There have been an increasing number of studies to explore the imaging data analysis methods for $\left[{ }^{18} \mathrm{~F}\right]$ DPA-714 $4^{43-45}$. Compartmental analysis is the gold standard for parameter estimation, however, this method is hard to perform in rodents since arterial blood sampling throughout the dynamic PET scan is difficult. Some radiotracers can use a reference region to estimate the binding parameters, but there is no suitable reference brain region for TSPO PET ${ }^{46}$. At present, the quantification for $\left[{ }^{18} \mathrm{~F}\right] \mathrm{DPA}-714$ is to calculate SUV, SUV ratio $(\mathrm{SUVr})$, or $\% \mathrm{ID} / \mathrm{g}^{20}$, and the parameter estimation can be performed by using cerebellum gray matter (GM) as a pseudo-reference region ${ }^{43}$. However, the quantitative imaging results in this study did not seem to support the use of GM as an ideal reference region. Therefore, we did not use SUVr to substitute SUV as the primary indicator. A better way to reflect the specific uptake of the radiotracer in the brain is to conduct parametric analysis to calculate total distribution volume $\left(\mathrm{V}_{\mathrm{T}}\right)$ or $\mathrm{BP}_{\mathrm{ND}}$. In this study, we calculated $\mathrm{BP}_{\mathrm{ND}}$ using $\mathrm{GM}$ as the pseudoreference region $\left(\mathrm{BP}_{\mathrm{ND}}-\mathrm{CBL}\right)$. Our blocking experiments validated the specific binding of $\left[{ }^{18} \mathrm{~F}\right] \mathrm{DPA}-714$ as reported in previous studies ${ }^{36}$. We tried to use the data obtained in the blocking experiments as pseudo-reference regions to calculate $\mathrm{BP}_{\mathrm{ND}}\left(\mathrm{BP}_{\mathrm{ND}}-\mathrm{BLO}\right)$. The results showed that there were significant correlations between $\mathrm{BP}_{\mathrm{ND}}-\mathrm{CBL}$, $\mathrm{BP}_{\mathrm{ND}}-\mathrm{BLO}$, and SUV $(p<0.001)$. Whether this method is reliable enough for parameter estimation need to be further validated using compartmental analysis. It should be noted that the use of blocking data to estimate the parameters is a calculation between two dynamic scans. Therefore, it is necessary to strictly control the same scan conditions and normalize the body weights and injection doses of the rats.

Recently, the antidepressant effects of GLP-1R agonists were proved $^{27,47}$. A meta-analysis indicated that GLP-1 analogs resulted in a significant reduction of depression rating $\mathrm{scales}^{28}$. Besides, preclinical studies found that GLP-1 analogs and some other medications can modulate the GLP-1R expression in the brain ${ }^{29}$. However, there are few noninvasive methods available to assess the GLP-1R expression in the brain in vivo. Our previous study demonstrated that PET combined with radiolabeled exendin-4, a widely used GLP-1 analog, can be used for GLP-1R detection in vivo ${ }^{32}$. In this study, we evaluated the brain GLP-1R expression using ${ }^{18} \mathrm{~F}$-labeled exendin-4 and the PET imaging results immediately after the CUMS modeling showed no significant differences in brain GLP$1 \mathrm{R}$ expression between CUMS group and control group. However, the quality of parametric images of $\left[{ }^{18} \mathrm{~F}\right]$ exendin-4 PET was limited due to the low brain uptake and fast washout of the tracer showed in the dynamic results. The permeability of $\left[{ }^{18} \mathrm{~F}\right]$ exendin- 4 to cross the BBB is not ideal and the brain GLP-1R expression might be low, therefore, the quantification of imaging data would be essential. The blocking experiments in this study showed a slight but significant difference between blocking and unblocking groups which validated the specificity of this tracer in the brain. Then we did parameter estimation for $\left[{ }^{18} \mathrm{~F}\right]$ exendin-4. Similar to $\left[{ }^{18} \mathrm{~F}\right] \mathrm{DPA}-714$, the results indicated that cerebellum could not be an ideal true reference region. The definite expression pattern of brain GLP-1R expression is still unclear and its widespread expression might make it difficult to find a suitable reference region which is also similar to $\left[{ }^{18} \mathrm{~F}\right] \mathrm{DPA}-714$.

Light therapy has been well accepted for the treatment of depression. In recent years, studies using $\left[{ }^{11} \mathrm{C}\right]$ harmine, $\left[{ }^{11} \mathrm{C}\right] \mathrm{DASB}$, and $\left[{ }^{18} \mathrm{~F}\right] \mathrm{FDG}$ PET proved that light therapy can modulate the levels of MAO-A, 5-HTT, and glucose metabolism in the brain of depression patients ${ }^{6,7,48}$. On the one hand, these studies provided useful information for understanding light therapy. On the other hand, they also proved that the noninvasive PET imaging has unique value and importance in monitoring pathological progress and guiding clinical medications of neuropsychiatric disorders. In this study, we used $\left[{ }^{18} \mathrm{~F}\right] \mathrm{DPA}-714$ to evaluate the neuroinflammatory changes in the brain of CUMSinduced depression rats during light therapy. The results indicated that light therapy could not improve neuroinflammation caused by CUMS. The binding affinity and specificity of $\left[{ }^{18} \mathrm{~F}\right]$ exendin- 4 used in this study to GLP-1R have been validated previously in neuroendocrine tumors and pancreatic $\beta$-cell tracking ${ }^{37,49}$. Here, we used this tracer to evaluate the changes of brain GLP-1R expression during light therapy, and the results suggested that brain GLP-1R expression of untreated rats might continue to decrease within 5 weeks, while light therapy prevented the reduction. However, these results should be carefully considered since the brain uptake of $\left[{ }^{18} \mathrm{~F}\right]$ exendin- 4 is relatively low and no suitable quantification method has been established. We are currently doing more work to improve the efficiency of $\left[{ }^{18} \mathrm{~F}\right]$ exendin- 4 crossing the blood-brain barrier (BBB), so as to get more convincing quantification data and visualization performance.

This study had some limitations. For parameter estimation, we did not perform compartmental analysis because it was difficult to continuously gather blood samples during dynamic PET scans. However, SUV has also been validated as a promising indicator for PET imaging data quantification. The characteristics of $\left[{ }^{18} \mathrm{~F}\right]$ exendin -4 for brain imaging need to be improved. We 
have compared several ${ }^{18} \mathrm{~F}$-labeled exendin- 4 and found that $\left[{ }^{18} \mathrm{~F}\right] \mathrm{FBEM}-\mathrm{Cys}^{39}$-exendin-4 have higher BBB permeability than $\left[{ }^{18} \mathrm{~F}\right]$ AlF-NOTA-MAL-Cys ${ }^{39}$-exendin- $4{ }^{50}$. However, the radiosynthesis of $\left[{ }^{18} \mathrm{~F}\right] \mathrm{FBEM}-\mathrm{Cys}^{39}$-exendin- 4 is much more complicated than $\left[{ }^{18} \mathrm{~F}\right]$ AlF-NOTAMAL-Cys ${ }^{39}$-exendin- 4 and its yield is low ${ }^{51}$. Therefore, we think that $\left[{ }^{18} \mathrm{~F}\right] \mathrm{FBEM}-\mathrm{Cys}{ }^{39}$-exendin-4 might not be suitable for longitudinal dynamic PET scans of brain imaging. The high yield and simple synthesis of $\left[{ }^{18} \mathrm{~F}\right] \mathrm{AlF}-$ NOTA-MAL-Cys ${ }^{39}$-exendin- 4 make it possible to perform multiple PET scans in a short period. Another point should be noted is that since the amount of imaging data is too large and the sensitivity of PET has a limit, the interpretation of the statistical significance needs to be cautious although we have conducted correction. In the Supplementary Tables, we list the SUV and their variances of all tracers in all regions at all time points, so as to make the display of the results more cautious and objective. Finally, a common limitation of all TSPO PET studies is that microglia have both pro-inflammatory and anti-inflammatory phenotypes, however, TSPO PET has limited ability to differentiate microglia between different phenotypes ${ }^{52}$.

In conclusion, we validated the widespread microglial activation and reduced glucose metabolism in the brain of depressive rats using PET imaging. Light therapy can ameliorate the depressive-like behavior, reverse the reduced glucose metabolism, and prevent the decline of GLP-1R expression. However, light therapy has no amelioration for the microglial activation in depression. Besides, the $\left[{ }^{18} \mathrm{~F}\right] \mathrm{DPA}-714$ and $\left[{ }^{18} \mathrm{~F}\right]$ exendin-4 PET showed quantitative potential in brain imaging, and these applications would be feasible and valuable for a better understanding of the pathophysiological process of depression and the clinical translational research in this field.

\section{Acknowledgements}

This work was supported by National Key Research and Development Program of China (2016YFC1306600), National Significant New Drugs Creation Program (2017ZX09304021), National Natural Science Foundation (31671035), Jiangsu Provincial Medical Innovation Team (CXTDA2017024), and Wuxi International $R$ \& D cooperation projects (WX0303B010518180012PB).

\footnotetext{
Author details

${ }^{1} \mathrm{NHC}$ Key Laboratory of Nuclear Medicine, Jiangsu Key Laboratory of Molecular Nuclear Medicine, Jiangsu Institute of Nuclear Medicine, Wuxi 214063 Jiangsu, China. ${ }^{2}$ Peking-Tsinghua Center for Life Sciences, Peking University, 100871 Beijing, China. ${ }^{3}$ Peking University Sixth Hospital, Peking University Institute of Mental Health, NHC Key Laboratory of Mental Health (Peking University), National Clinical Research Center for Mental Disorders (Peking University Sixth Hospital), Peking University, 100191 Beijing, China
}

\section{Conflict of interest}

The authors declare that they have no conflict of interest.

\section{Publisher's note}

Springer Nature remains neutral with regard to jurisdictional claims in published maps and institutional affiliations.
Supplementary Information accompanies this paper at (https://doi.org/ 10.1038/s41398-020-01155-z).

Received: 30 July 2020 Revised: 27 November 2020 Accepted: 3 December 2020

Published online: 06 January 2021

\section{References}

1. Wirz-Justice, A. et al. Brightening depression. Science 303, 467-469 (2004).

2. Videnovic, A. et al. Timed light therapy for sleep and daytime sleepiness associated with Parkinson disease: a randomized clinical trial. JAMA Neurol. 74, 411-418 (2017)

3. van, Maanen, A., Meijer, A. M., van, der, Heijden, K. B. \& Oort, F. J. The effects of light therapy on sleep problems: a systematic review and meta-analysis. Sleep Med. Rev. 29, 2952-2962 (2016).

4. Sit, D. K. et al. Adjunctive bright light therapy for bipolar depression: a randomized double-blind placebo-controlled trial. Am. J. Psychiatry 175, 131-139 (2018).

5. Martorell, A. J. et al. Multi-sensory gamma stimulation ameliorates Alzheimer'sassociated pathology and improves cognition. Cell 177, 256-271 (2019).

6. Spies, M. et al. Brain monoamine oxidase $A$ in seasonal affective disorder and treatment with bright light therapy. Transl. Psychiatry 8, 198 (2018).

7. Tyrer, A. E. et al. Serotonin transporter binding is reduced in seasonal affective disorder following light therapy. Acta Psychiatr. Scand. 134, 410-419 (2016).

8. Malhi, G. S. \& Mann, J. J. Depression. Lancet 392, 2299-2312 (2018).

9. Setiawan, E. et al. Role of translocator protein density, a marker of neuroinflammation, in the brain during major depressive episodes. JAMA Psychiatry 72, 268-275 (2015)

10. Leu, S. J., Shiah, I. S., Yatham, L. N., Cheu, Y. M. \& Lam, R. W. Immuneinflammatory markers in patients with seasonal affective disorder: effects of light therapy. J. Affect. Disord. 63, 27-34 (2001).

11. Song, $C$. et al. Enhanced inflammatory and T-helper-1 type responses but suppressed lymphocyte proliferation in patients with seasonal affective disorder and treated by light therapy. J. Affect. Disord. 185, 90-96 (2015).

12. Vivash, L. \& O'Brien, T. J. Imaging microglial activation with TSPO PET: lighting up neurologic diseases? J. Nucl. Med. 57, 165-168 (2016).

13. Hannestad, J. et al. The neuroinflammation marker translocator protein is not elevated in individuals with mild-to-moderate depression: a [11C]PBR28 PET study. Brain. Behav. Immun. 33, 33131-33138 (2013).

14. Su, L. et al. Neuroinflammatory and morphological changes in late-life depression: the NIMROD study. Br. J. Psychiatry 209, 525-526 (2016).

15. Holmes, S. E. et al. Elevated translocator protein in anterior cingulate in major depression and a role for inflammation in suicidal thinking: a positron emission tomography study. Biol. Psychiatry 83, 61-69 (2018).

16. Li, H., Sagar, A. P. \& Keri, S. Translocator protein (18kDa TSPO) binding, a marker of microglia, is reduced in major depression during cognitive-behavioral therapy. Prog. Neuropsychopharmacol. Biol. Psychiatry 83, 1-7 (2018).

17. Setiawan, E. et al. Association of translocator protein total distribution volume with duration of untreated major depressive disorder: a cross-sectional study. Lancet Psychiatry 5, 339-347 (2018)

18. Chauveau, F. et al. Comparative evaluation of the translocator protein radioligands 11C-DPA-713, 18F-DPA-714, and 11C-PK11195 in a rat model of acute neuroinflammation. J. Nucl. Med. 50, 468-476 (2009).

19. Wang, Y. et al. [18F]DPA-714 PET imaging of AMD3100 treatment in a mouse model of stroke. Mol. Pharm. 11, 3463-3470 (2014).

20. Nguyen, D. L. et al. Longitudinal positron emission tomography imaging of glial cell activation in a mouse model of mesial temporal lobe epilepsy: toward identification of optimal treatment windows. Epilepsia 59, 1234-1244 (2018).

21. Hamelin, L. et al. Distinct dynamic profiles of microglial activation are associated with progression of Alzheimer's disease. Brain 141, 1855-1870 (2018).

22. Wang, Y. L. et al. Microglial activation mediates chronic mild stress-induced depressive- and anxiety-like behavior in adult rats. J. Neuroinflammation 15, 21 (2018).

23. Bruce-Keller, A. J., Salbaum, J. M. \& Berthoud, H. R. Harnessing gut microbes for mental health: getting from here to there. Biol. Psychiatry 83, 214-223 (2018).

24. Everard, A. \& Cani, P. D. Gut microbiota and GLP-1. Rev. Endocr. Metab. Dis. 15, 189-196 (2014) 
25. Huang, X. F. \& Song, X. Effects of antipsychotic drugs on neurites relevant to schizophrenia treatment. Med. Res. Rev. 39, 386-403 (2019).

26. Lopez, M., Nogueiras, R., Tena-Sempere, M. \& Dieguez, C. Hypothalamic AMPK a canonical regulator of whole-body energy balance. Nat. Rev. Endocrinol. 12 421-432 (2016).

27. Weina, $H$. et al. Liraglutide attenuates the depressive- and anxiety-like behaviour in the corticosterone induced depression model via improving hippocampal neural plasticity. Brain Res. 1694, 55-62 (2018).

28. Pozzi, M. et al. A systematic review of the antidepressant effects of glucagonlike peptide 1 (GLP-1) functional agonists: further link between metabolism and psychopathology. J. Affect. Disord. 257, 30593-2 (2019). S0165-0327(19).

29. Sun, J. et al. Clostridium butyricum attenuates chronic unpredictable mild stress-induced depressive-like behavior in mice via the gut-brain axis. J. Agr. Food Chem. 66, 8415-8421 (2018).

30. Zhao, Y. et al. Geniposide improves repeated restraint stress-induced depression-like behavior in mice by ameliorating neuronal apoptosis via regulating GLP-1R/AKT signaling pathway. Neurosci. Lett. 676, 19-26 (2018).

31. Cork, S. C. et al. Distribution and characterisation of Glucagon-like peptide-1 receptor expressing cells in the mouse brain. Mol. Metab. 4, 718-731 (2015).

32. Wang, L. et al. Age-related change of GLP-1R expression in rats can be detected by [18F]AIF-NOTA-MAL-Cys39-exendin-4. Brain Res. 1698, 213-219 (2018).

33. Laforce, R. et al. Molecular imaging in dementia: past, present, and future. Alzheimer Dement. 14, 1522-1552 (2018).

34. Leng, L. et al. Menin deficiency leads to depressive-like behaviors in mice by modulating astrocyte-mediated neuroinflammation. Neuron 100, 551-563.e7 (2018).

35. Huang, L. et al. A visual circuit related to habenula underlies the antidepressive effects of light therapy. Neuron 102, 128-142 (2019).

36. James, M. L. et al. DPA-714, a new translocator protein-specific ligand: synthesis, radiofluorination, and pharmacologic characterization. J. Nud. Med. 49, 814-822 (2008).

37. $\mathrm{Xu}, \mathrm{Q}$. et al. Preliminary evaluation of [18F]AIF-NOTA-MAL-Cys39-exendin-4 in insulinoma with PET. J. Drug Target 23, 813-820 (2015).

38. Brainard, G. C. et al. Short-wavelength enrichment of polychromatic light enhances human melatonin suppression potency. J. Pineal Res. 58, 352-361 (2015).
39. Leonard, B. E. Inflammation and depression: a causal or coincidental link to the pathophysiology? Acta Neuropsychiatr. 30, 1-16 (2017).

40. Villemagne, V. L., Dore, V., Burnham, S. C., Masters, C. L. \& Rowe, C. C. Imaging tau and amyloid-beta proteinopathies in Alzheimer disease and other conditions. Nat. Rev. Neurol. 14, 225-236 (2018).

41. Ge, J., Zhang, Q., Zeng, J., Gu, Z. \& Gao, M. Radiolabeling nanomaterials for multimodality imaging: New insights into nuclear medicine and cancer diagnosis. Biomaterials 228, 119553 (2020).

42. Wimberley, C. et al. Longitudinal mouse-PET imaging: a reliable method for estimating binding parameters without a reference region or blood sampling. Eur. J. Nucl. Med. Mol. Imaging 47, 2589-2601 (2020).

43. Golla, S. S. et al. Parametric binding images of the TSPO Ligand 18F-DPA-714. J. Nucl. Med. 57, 1543-1547 (2016).

44. Van, Camp, N. et al. Assessment of simplified methods for quantification of [18F]-DPA-714 using 3D whole-brain TSPO immunohistochemistry in a nonhuman primate. J. Cereb. Blood Flow. Metab. 40, 1103-1116 (2020).

45. Golla, S. S. et al. Quantification of [18F]DPA-714 binding in the human brain: initial studies in healthy controls and Alzheimer's disease patients. J. Cereb. Blood Flow. Metab. 35, 766-772 (2015).

46. Wimberley, C. et al. Impact of endothelial 18-kDa translocator protein on the quantification of 18F-DPA-714. J. Nucl. Med. 59, 307-314 (2018).

47. Kahal, H., Kilpatrick, E., Rigby, A., Coady, A. \& Atkin, S. The effects of treatment with liraglutide on quality of life and depression in young obese women with PCOS and controls. Gynecol. Endocrinol. 35, 142-145 (2019).

48. Kohno, K. et al. Postcomparison of [18F]-fluorodeoxyglucose uptake in the brain after short-term bright light exposure and no intervention. Acta Psychiatr. Scand. 134, 65-72 (2016).

49. Mi, B. et al. Non-invasive glucagon-like peptide-1 receptor imaging in pancreas with 18F-Al labeled Cys39-exendin-4. Biochem. Biophys. Res. Commun. 471, 47-51 (2016).

50. Liu, Y. et al. Multi-target PET evaluation in APP/PS1/tau mouse model of Alzheimer's disease. Neurosci. Lett. 728, 134938 (2020).

51. $\mathrm{Xu}, \mathrm{Y}$. et al. Insulinoma imaging with glucagon-like peptide-1 receptor targeting probe 18F-FBEM-Cys39-exendin-4. J. Cancer Res. Clin. Oncol. 140, 1479-1488 (2014).

52. Rupprecht, R. et al. Translocator protein ( $18 \mathrm{kDa})(\mathrm{TSPO})$ as a therapeutic target for neurological and psychiatric disorders. Nat. Rev. Drug Discov. 9, 971-988 (2010). 